\title{
Development of a Seed Analyzer using the Techniques of Computer Vision
}

\author{
Sandeep Arya and Parveen Lehana \\ Department of Physics and Electronics, University of Jammu, Jammu, India \\ Email: snp09arya@gmail.com
}

\begin{abstract}
Seed analyzer is a mechatronic system for differentiating different types of seeds. It has enormous applications in agriculture industry. Although several seed analyzers are available in the market, still there is large scope for improvement because of their various disadvantages. Most of the analyzers are built using very simple image processing techniques and electronic principles. In this research paper, the proposed idea is to develop the method of identifying the best quality seeds by using the techniques of computer vision. Analysis of the resultant images has shown that the quality of the processed image using the proposed technique is improved considerably. The task of matching of the processed image with the stored database of the seeds is on our future work.
\end{abstract}

Keywords:

Seed analyzer, pixel, computer vision, image processing, image.

\section{Introduction:}

Image processing is a stimulating and an active field of research, where disciplines such as Engineering, Computer Science, Physics, Biology and Medicine inter-disciplinarily co-operate in order to improve health care. In this regard, medical images are the basis of diagnostics, treatment planning, and treatment, but medical images are likewise important for medical education, research and epidemiology [1] [2]. A seed analyzer is a guide to understand the qualities of grains or seeds and provides an introduction to the analysis of seeds in a clear and concise format. In literature, basic information about the seed analyzers has been presented by several authors onstandardized testing procedures for seed quality characteristics as well as dough properties. The outcome by processing the tests can explain the performance a product quality. Many laboratories testing of a variety of seeds are used to evaluate the seed quality. The seed analyzers are the model systems that may be used to predict commercial production for common uses of seeds and grains worldwide. The picture taken from the low resolution cameras may not provide a good image of the seed but by using the technique of computer vision, the quality of image can gets enhanced. The results from these processes may prevent the adulteration of seed lots and protect the consumer from buying the seed through fraudulent representation. The image processing techniques has many applications as it includes digital cameras, intelligent traffic monitoring, handwriting recognition on checks, signature validation and so on. However, it is not uncommon that images are contaminated by various noises and distortions due to several unavoidable reasons. Poor image sensors, imperfect instruments, problems with data acquisition process, transmission errors and interfering natural phenomena are its main sources. Therefore, it is obligatory to detect and remove noises present in the images [3]. In past decades, attention has been focusedon monochrome image segmentation 
whose goal is to separate individual objects in the perception of the scene. A common problem in segmentation of monochrome image occurs when an image has a background of varying gray levels such as gradually changing shades [4]. This problem is inherent, since intensity is the only available information from monochrome images. It has long been recognized that the human eye can sense only in the neighborhood of one or two dozen intensity levels at any one point in a complex image due to its brightness adaptation but can discern thousands of color shades and intensities [5]. Color image segmentation techniques can be roughly classified into four types such as histogram based approaches, neighborhood based approaches, clustering based approaches and hybrid based approaches. Gray-level thresholding is one of the oldest techniques for image segmentation [6]. Threshold may be chosen based onhistogram [7] or on gray-level co-occurrence matrix [8], or by analyzing intra-region and inter-region homogeneity [9]. Histogram thresholding is a popular technique that looks for the peaks and valleys in histogram [10] [11]. It assumes that images are composed of regions with different gray level ranges. The histogram of an image can be separated into a number of peaks (modes), each corresponds to one region, and there exists a threshold value corresponding to the valley between two adjacent peaks. The most important advantage of this technique deceit is its simple computation [11]. In this experiment, our idea is only to develop the process of recognition of seeds using the techniques of image processing and computer vision. By using this technique we can visualize the above processes using camera and the computer. The idea surrounds near the execution of a well-known technique, "mathematical morphology" used in image processing and computer vision [12] [13] [14]. In this, the shape oriented approach treats the image as one set and the kernel of operation, commonly known as structuring element (SE), as another set. Different standard morphological operations namely dilation, erosion, opening, closing etc. are basically set-theoretic operations between these two sets. The shape and the size of the SE plays an important role in detecting or extracting features of given shape and size from the image. Applications of mathematical morphology in gray-level image segmentation can be found in [15] [16] [17].

In this paper, the proposed idea is only to develop an algorithm for analyzing seeds using an image processing techniques of computer vision. The algorithm detects the suitable segments at every scale. Analysis of the resultant images has shown that the quality of the processed image using the proposed technique is improved considerably. The methodology employed for our investigations is described in Section 2. Results and conclusions are discussed in Section 3 and 4.

\section{METHODOLOGY:}

In this paper our proposed method has been divided into four subparts. The output obtained from one part is taken as input to the next part. This can be represented by the subsequent work flow graph as shown in fig. 1.

\subsection{Morphological Opening to Estimate the Background}

This is pre-processing step which is required to produce better results. The input image is to be adjusted for proper processing. The background of the image has to be in contrast of the main image. Following steps are involved in this process.

(a) First of all the colored mage is to be converted into grayscale image.

(b) Subtract background image from original image,

(c) Adjusting the contrast of the image. 


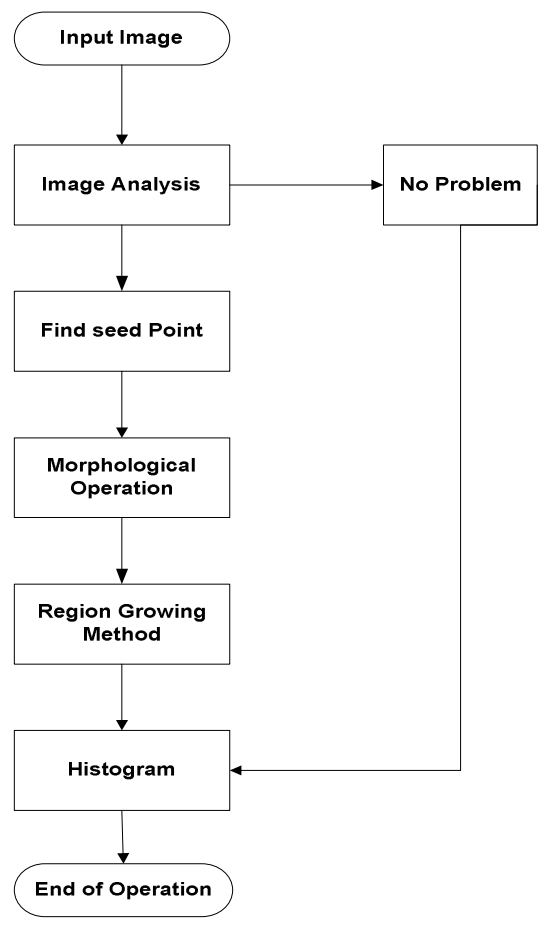

Fig. 1: Flow chart showing seed analysis by image processing method.

\subsection{Seed point selection}

The image obtained after adjusting contrast is taken as input in this part of the project. For the seed point selection, the following steps are performed:

(a) Convert the obtained gray image into the binary image.

(b) Find maximum length and breadth of the seed

(c) Finding the edges of the binary image.

(d) Complementing the image.

\subsection{Morphological Image Enhancement}

Morphology mainly deals with the contour and structure of the object. So this is used to perform object extraction, noise removal procedure etc [18]. In this regard, we are applying these operations in this step to enhance the object boundary and to remove the noise from the image. As discussed in introduction, the most basic morphological operations are dilation and erosion. Dilation adds pixels to the boundaries of objects in an image, while erosion removes pixels on object boundaries. The number of pixels added or removed from the objects in an image depends on the size and shape of the structuring element used to process the image. In the morphological dilation and erosion operations, the state of any given pixel in the output image is determined by applying a rule to the corresponding pixel and its neighbors in the input image. The rule used to process the pixels defines the operation as dilation or erosion. The most important part in the morphological operation is to choose the structuring element. A structuring element is a matrix consisting of only 0's and 1's that can have any arbitrary shape and size. The pixels describe the relative presence of one level in the neighborhood of another level in a texture of image. The pixels with value of 1 define the neighborhood. Thus, a neighborhood element is used to calculate the relative presence of one level with respect to 
International Journal of Distributed and Parallel Systems (IJDPS) Vol.3, No.1, January 2012

another. Two-dimensional, or flat, structuring elements are typically much smaller than the image being processed. The centre value of the structuring element, called the origin, identifies the pixel of interest. The pixels in the structuring element containing 1's define the neighborhood of the structuring element. In our research, we are taking structuring element of 3 x 3 matrix shape. In the operation of image dilation and erosion we are considering structuring element of different matrices, so that the image obtained is free from small unwanted parts (distortions). In MATLAB working environment there are two built in functions used for dilation and erosion.These morphological functions point the origin of structuring element, its center element over the pixel of interest is in the input image. For pixels at the border of the image, parts of the neighborhood defined by the structuring element can extend past the border of the image. To process border pixels, the morphological functions assign a value to these undefined pixels, as if the functions had padded the image with additional rows and columns. The value of these padding pixels varies for dilation and erosion operations.

\subsection{Seeded Region Growing Method}

The results so obtained after morphological operation is taken as input in this stage. This approaches towards segmentation that examines neighboring pixels of initial "seed points" and determines whether the pixel neighbors should be added to the region. The whole process has been processed using the proper algorithm with the help of image processing. The most important purpose of segmentation is to partition an image into regions. Some specific segmentation methods such as "Thresholding" used to achieve this goal by looking for the boundaries between regions based on discontinuities in gray levels or color properties [19]. Region-based segmentation is a technique for determining the region directly.

The basic formulation for region- based segmentation is

$$
\sum \mathbf{R}_{\mathrm{i}}=\mathbf{R}_{\mathbf{0}} \text { where } \mathbf{i}=\mathbf{1}, \mathbf{2}, 3, \ldots \ldots \ldots \ldots, \mathrm{n}
$$

means the segmentation must be complete and every pixel must be in the region. Here $\mathrm{R}_{\mathrm{i}}$ is a connected region.

$$
\mathbf{R}_{\mathrm{i}} \cap \mathbf{R}_{\mathrm{j}}=\boldsymbol{\Phi}
$$

The above equation means the regions mustbe disjoint, so that a clear separation from each other can be identified.

\section{Results}

We have implemented our work using MATLAB 7.6.0.324 and the results for a few set of images are included in the table 1 . The table shows the output results taken after processing the input image taken by camera using proper algorithm. The algorithm helps in analyzing the seeds and the output images taken is shown in fig 2.

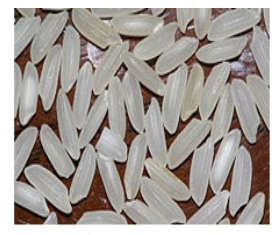

A

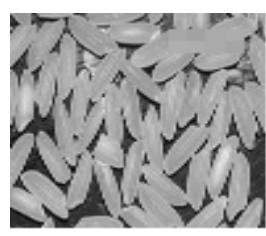

B

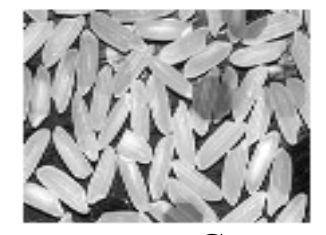

$\mathrm{C}$

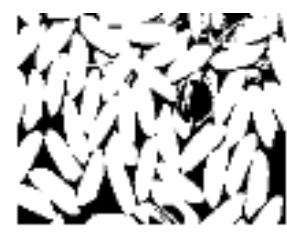

$\mathrm{D}$ 
International Journal of Distributed and Parallel Systems (IJDPS) Vol.3, No.1, January 2012

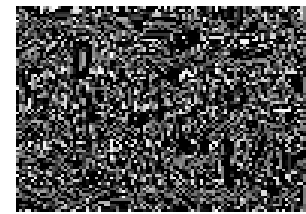

$\mathrm{E}$

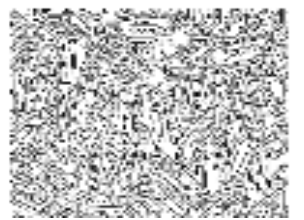

$\mathrm{F}$

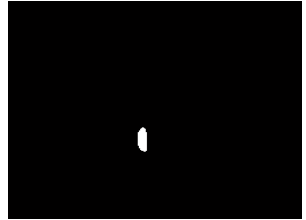

G

Fig. 2: Output images for different results

Table 1: Results for different images.

\begin{tabular}{|c|c|c|}
\hline S.No. & Image No. & Results \\
\hline 1. & A & Input Image \\
\hline 2. & B & Grayscale Image \\
\hline 3. & C & Image after subtracting background \\
\hline 4. & D Image \\
\hline 5. & E & Complemented Image \\
\hline 6. & F & Seed having smallest Area \\
\hline 7. & G & \\
\hline
\end{tabular}

The number of seeds has been counted using an algorithm and it comes out to be 22 well defined seeds. The average area of a single seed has been calculated and it comes out to be 335 pixels. The seed occupying minimum area is also calculated and it comes out to be 5 pixels having index number 7 .

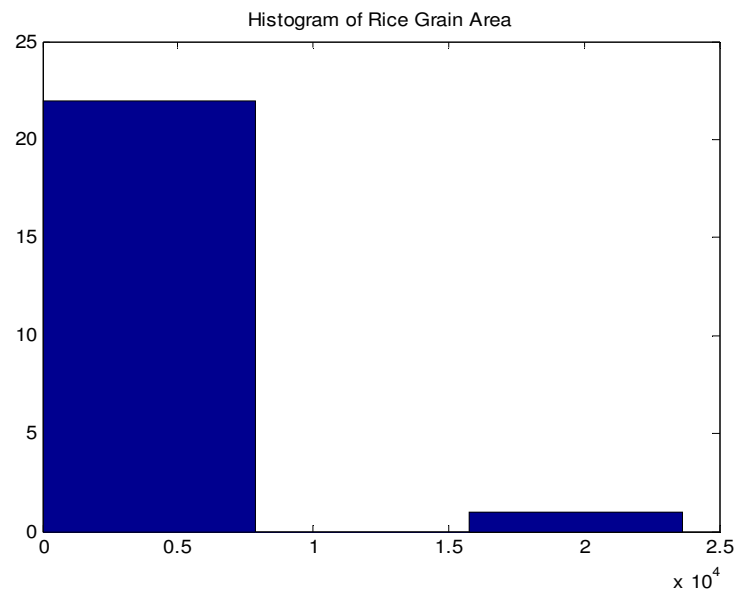

Fig. 3: Histogram for the resultant seeds. 
International Journal of Distributed and Parallel Systems (IJDPS) Vol.3, No.1, January 2012

The histogram is created as shown in figure 3 and it shows that 22 seeds having the same size whereas other 3 seeds has different or very small size in comparison to others as shown in the graph 1.

\section{Conclusion and Future work}

This method is economical than other seed analyzers and can be easily implemented with great accuracy.It does not rely on massively looping algorithms, making it more efficient. It is possible to determine the average area of the grain easily and can accurately count the number of grains in the image taken. We can enhance the quality of the image. We proposed a new, robust, fast and fully automatic algorithm. The algorithm needs no prior information or training process. We successfully find the seed points and the segmentation results obtained are very much accurate. There are only a small amount of pixels which are misclassified. So we can say that this method gives better results compared to other methods.

The future work is the next stage of analyzing the seed and isolating the impurity from the seed lot with the help of robotic hand and microcontroller. The expected output will be the best quality seed by taking out the impurities and the impure or the seeds in parts. This will help the agriculture industries in rectifying the quality of seed and will save the time and labor. The future work also includes the reduction of the total execution time so that along with good result the execution time can be reduced [20]. This technique will also provide the customers or users, the best quality products from the agricultural industries.

\section{References}

[1] Thomas M. Deserno, Til Aach, Katrin Amunts, Walter Hillen, Torsten Kuhlen, Ingrid Scholl, "Advances in medical image processing", Journal of Computer Sci Res Dev, ㄷ Springer-Verlag 2010

[2] Deserno TM, Handels H, Meinzer HP, Tolxdorff T (eds) (2010), "Bildverarbeitungfür die Medizin 2010", Algorithmen Systeme Anwendungen, Springer, Berlin

[3] Tanzila Saba, Amjad Rehman and Ghazali Sulong, "An intelligent approach to image denoising”, Journal of Theoretical and Applied Information Technology, () 2005 -2010, JATIT \& LLS.

[4] A. Buades, B. Coll, and J. Morel, "Nonlocal image and movie denoising", International Journal of Computer Vision, Vol. 76, No. 2, 2008, PP. 123-139.

[5] M. Mahmoudi and G. Sapiro, "Fast image and video denoising via nonlocal means of similar neighborhoods", IEEE Signal Processing Letters, Vol. 12, No. 12, 2005, pp. 839-842.

[6] A. Rosenfeld and A. C. Kak, “Digital Picture Processing”, 2nd ed. New York: AP, 1982, vol. 1/2.

[7] B. Chanda, B. B. Chaudhuri, and D. Dutta Majumder, "A modified scheme for segmenting noisy images,” IEEE Trans. Syst., Man, Cybern., vol. 18, no. 3, pp. 458-467, 1988.

[8] B. Chanda and D. Dutta Majumder, "A note on use of gray-level co-occurrence matrix in threshold selection,” Signal Process, vol. 15, no. 2, pp. 149-167, 1988.

[9] J. S. Weszka and A. Rosenfeld, "Threshold evaluation techniques," IEEE Trans. Syst., Man, Cybern., vol. SMC-8, pp. 622-629, 1978.

[10] P. K. Sahoo, S. Soltani, A. K C. Wong, and Y.C. Chen, "A survey on thresholding techniques," Computer Vision Graphics Image Processing, vol. 41, 1988, pp. 233-260.

[11] Kanchan Deshmukh, Ganesh Shinde, "Adaptive Color Image Segmentation Using Fuzzy MinMax Clustering”, Engineering Letters, 13:2, EL_13_2_2 (Advance online publication: 4 August 2006)

[12] G. M. Matheron, "Random Sets and Integral in Geometry”, New York: Wiley, 1975.

[13] J. Serra, "Image Analysis Using Mathematical Morphology", London, U.K.: Academic, 1982.

[14] R. M. Haralick and L. G. Shapiro, "Computer and Robot Vision", Reading, MA: AddisonWesley, 1992, vol. 1. 
International Journal of Distributed and Parallel Systems (IJDPS) Vol.3, No.1, January 2012

[15] P. T. Jackway, "Gradient watershed in morphological scale-space", IEEE Trans, Image Processing, vol. 5, pp. 913-921, 1996.

[16] P. Salembier and J. Serra, "Morphological multiscale image segmentation," in Proc. Visual Communication and Image Processing, Boston, MA, 1992, pp. 620-631.

[17] P. Salembier, "Morphological multiscale segmentation for image coding," Signal Process, vol. 38, pp. 359-386, 1994.

[18] U. sivarajan, K J Jayapragasam, Y F Abdul Aziz, K Rahmat, "Dynamic contrast enhancement Magnetic resonanace imaging evaluation of Breast Lesions: A morphological and Quantitiveanalysis", J Hkcoll Radiol, 2009

[19] "Digital Image Processing", 3/E by Rafael C. Gonzalez, Richard E. Woods, ISBN-10: 013168728X

[20] Sukhvinder singh, Sukhbeer singh, "Parallel image processing concepts", IJCCT vol.1, August 2010. 